\title{
Medial Open-Wedge Supramalleolar Osteotomy for Patients with Takakura 3B Ankle Osteoarthritis: A Mid- to Long-Term Study
}

\author{
Yang $X u^{1}$ and Xiang-yang $X u \mathbb{D}^{2}$ \\ ${ }^{1}$ Ruijin Hospital North, Shanghai Jiao Tong University School of Medicine, Shanghai, China \\ ${ }^{2}$ Ruijin Hospital, Shanghai Jiao Tong University School of Medicine, Shanghai, China \\ Correspondence should be addressed to Xiang-yang Xu; xu664531@hotmail.com
}

Received 24 December 2018; Revised 17 April 2019; Accepted 26 May 2019; Published 17 June 2019

Academic Editor: Giuseppe Filardo

Copyright (C) 2019 Yang Xu and Xiang-yang Xu. This is an open access article distributed under the Creative Commons Attribution License, which permits unrestricted use, distribution, and reproduction in any medium, provided the original work is properly cited.

\begin{abstract}
It is controversial whether supramalleolar osteotomy is suitable for Takakura Stage 3B osteoarthritis or not. The aim of this study was to evaluate the outcomes of supramalleolar osteotomy in patients with Takakura 3B osteoarthritis. From February 2008 to August 2013, supramalleolar osteotomy was performed in 21 patients matching the inclusion criteria. The mean patient age at operation was $53.7 \pm 5.8$ years (range: 39 to 61 years). The mean duration of follow-up was $87.7 \pm 19.5$ months (range: 61 to 125 months). The radiologic evaluation included the tibial articular surface (TAS) angle, tibial lateral surface (TLS) angle, and talar tilt (TT) angle. Functional assessment was performed with use of the AOFAS, VAS, SF-36, and AOS. All patients were followed. TAS angle improved from $82.8 \pm 2.4$ to $90.3 \pm 2.3$. TLS angle changed from $77.5 \pm 2.8$ to $79.4 \pm 2.7$. The preoperative TT angle and postoperative TT angle were $13.4 \pm 3.6$ to $4.8 \pm 3.6$, respectively. For functional evaluation, the preoperative VAS and AOFAS-AH scores were $5.7 \pm 1.3$ and $48.0 \pm 15.8$, while the postoperative VAS and AOFAS-AH scores were $2.5 \pm 1.9$ and $74.8 \pm 11.5$. The mean SF-36 scale improved from $41.2 \pm 13.1$ to $66.7 \pm 14.9$. The AOS score improved from $61.4 \pm 12.5$ to $27.5 \pm 17.8$. 1 patient underwent total ankle replacement 3 years postoperatively. 4 patients remained stage 3B including the TAR one. 4 improved to stage $3 \mathrm{~A}, 11$ improved to stage 2 , and 2 improved to stage 1. Supramalleolar osteotomy combined with auxiliary procedures can restore the malalignment of ankle joint and modify the abnormal stress distribution so as to achieve functional improvement and improve radiographic stages.
\end{abstract}

\section{Introduction}

According to Takakura et al., varus ankle arthritis is classified into four stages in frontal view weight-bearing radiographs of ankle joint: 1 early sclerosis and formation of osteophytes without changing of ankle joint space; 2 narrowing of medial joint space without subchondral bone contact; 3 obliteration of ankle space with subchondral bone contact; 4 varus ankle joint with complete bone contact. And Stage 3 is divided into $3 \mathrm{~A}$ ankle joint obliteration limited to the medial malleolus and $3 \mathrm{~B}$ ankle joint obliteration was extended to the roof of the dome of talus $[1,2]$. Supramalleolar osteotomy is one of the joint-preserving methods for the treatment of varus type ankle arthritis, whereas the indications of this procedure are still controversial.
Joint-preserving procedures are still very important because many patients do not want to sacrifice the native joints. For varus type ankle osteoarthritis, supramalleolar osteotomy could restore the normal alignment of ankle and preserve the native ankle joint, which is particularly important for young patients with severe varus ankle osteoarthritis. Although supramalleolar osteotomy has been reported to result in substantial functional improvement and malalignment and achieve good outcomes, it was often recommended for early to midstage ankle arthritis (stage 2 or stage $3 \mathrm{~A}$ ) [1, 38]. But in our clinical practice, by performing supramalleolar osteotomy for stage $3 \mathrm{~B}$ ankle arthritis we did notice positive postoperative results.

This study hypothesized that supramalleolar osteotomy combined with auxiliary procedures such as calcaneal 


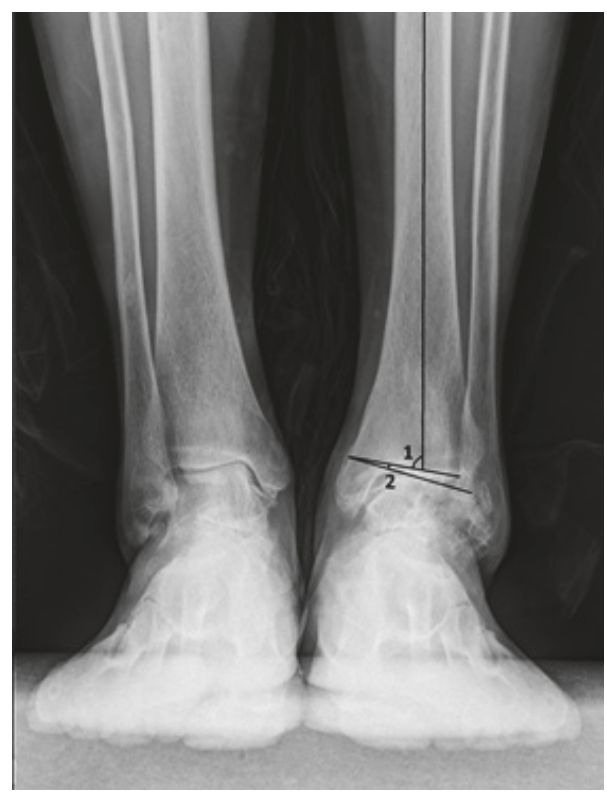

(a)

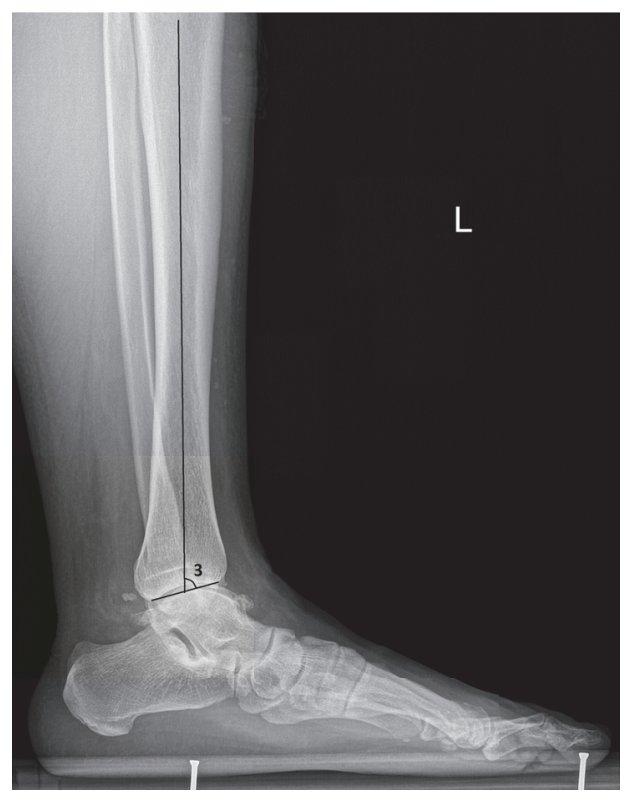

(b)

FIGURE 1: (a) 1: TAS: tibial articular surface angle, 2: TT: talar tilt angle. (b) 3: TLS: tibial lateral surface angle.

osteotomy and lateral ligament reconstruction are effective for the treatment of Takakura 3B ankle arthritis. It can restore the malalignment of ankle joint and modify the abnormal stress distribution so as to achieve functional improvement and reverse radiographic stages. The purpose of this study was to evaluate the radiographic and functional outcomes of supramalleolar osteotomy combined with auxiliary procedures for stage $3 \mathrm{~B}$ varus type ankle osteoarthritis.

\section{Patients and Methods}

This retrospective study was approved by institutional review board. From February 2008 to August 2013, supramalleolar osteotomy was performed in 21 patients who had symptomatic Takakura 3B ankle arthritis matching the inclusion criteria, including 3 men and 18 women. The mean patient age at operation was $53.7 \pm 5.8$ years (range: 39 to 61 years). The mean duration of follow-up was $87.7 \pm 19.5$ months (range: 61 to 125 months).

The inclusion criteria were (1) Takakura stage 3B ankle arthritis; (2) with clinical symptoms, such as walking pain and limitation of daily activities; (3) patients with follow-up time more than 5 years; (4) regional cartilage destruction on the medial side with more than $50 \%$ remaining cartilage on the lateral side in preoperative MRI scans.

The exclusion criteria were (1) end-stage ankle arthritis with global severe cartilage destruction on preoperative MRI scans and stage 1,2, or 3A ankle arthritis; (2) patients with neuropathic arthropathy or rheumatoid arthritis; (3) patients who had severe osteoporosis or large bone loss around ankle joint; (4) patients who had ankle surgery other than supramalleolar osteotomy like ankle joint distraction or osteotomy around ankle joint.
Standard weight-bearing radiographs of ankle joints were performed preoperatively, including anteroposterior (AP) and lateral views. The study also observed hindfoot varus deformity. It was recorded via hindfoot alignment view, which was described by Saltzman and el-Khoury [9]. In this study, preoperative MRI was also performed for each patient to see the damage situation of ankle joint cartilage. Supramalleolar osteotomy was performed for $3 \mathrm{~B}$ ankle arthritis with regional cartilage destruction on the medial side with more than $50 \%$ remaining cartilage on the lateral side. The radiologic evaluation included the tibial articular surface (TAS) angle, tibial lateral surface (TLS) angle, and talar tilt (TT) angle. In this study, the postoperative Takakura 1 ankle osteoarthritis was defined as TT angle $\leq 1^{\circ}$ without narrowing of ankle joint space (Figures 1(a) and 1(b)).

Functional assessment was performed with use of the American Orthopaedic Foot and Ankle Society anklehindfoot (AOFAS-AH) scale, the Visual Analogue Scale (VAS), the Short Form-36 (SF-36) scale, and the Ankle Osteoarthritis scale (AOS). Satisfactory results in this study were regarded as improvements to other stages, which were often accompanied with pain relief [10]. We think single scale especially AOFAS score cannot reflect the real situation of patients. So we combined several scales to assess the function of ankle joint.

2.1. Surgical Technique. A debridement of osteophytes around ankle joint was performed and soft tissue was released thoroughly especially the medial ligament first. The medial ligament was performed through a medial longitudinal approach. It was cut like a " $Z$ " shape to make sure the talus could return to normal site. The supramalleolar osteotomy was performed through a medial longitudinal 
approach. A K-wire was placed into the distal tibial about 4-5 $\mathrm{cm}$ above the ankle mortise to guide the osteotomy. The osteotomy was parallel to the tibial plafond. The study used structural allograft to fulfill the supramalleolar osteotomy. Intraoperative visualization and fluoroscopy were used to assess the adequacy of correction of distal tibial varus deformity. The study aimed to make TAS angle in neutral position or a little overcorrected but TAS angle was no more than $95^{\circ}\left(90^{\circ}-95^{\circ}\right)$ [11]. Then the osteotomy was fixed with a plate and interlocking screws. The foot was pushed intraoperatively to simulate weight-bearing situation to see whether the released talus could return to normal. In this position, the medial ligament was sutured. If there was laxity of lateral ligament, an augment procedure or reconstruction procedure was performed $[12,13]$.

The correction was assessed with the use of fluoroscopy. If it still remained residual hindfoot deformity after supramalleolar osteotomy, then the calcaneal osteotomy was performed. For patients had residual hindfoot deformity, a calcaneal lateral sliding osteotomy was performed to keep hindfoot a little valgus $\left(<5^{\circ}\right)$.

2.2. Postoperative Management. A cast was used for 6 weeks. Part weight bearing was allowed 6 weeks after operation with the cast. Full weight bearing was allowed two to three months after operation. Patients were required to go to outpatient at 6 and 8 weeks after operation and take weight-bearing radiographs. Patients came to outpatient 3 months, 6 months, and 1 year after operation to estimate the function of ankle. Then, patients came to hospital annually to take radiographs and estimate the function of ankle joints.

2.3. Statistical Analysis. All analyses were performed with SAS software version 8.1 (SAS Institute Inc, Cary, North Carolina). Descriptive statistics were calculated as mean \pm standard deviation. The paired Student $t$ test was adopted to compare the preoperative and postoperative radiographic measurements and AOFAS-AH, SF-36, AOS, and VAS scores. The significance level was set at $\mathrm{P}<0.05$.

\section{Results}

There was no loss of follow-up. Among the 21 patients, 9 patients underwent calcaneal osteotomies and 12 underwent lateral ligament reconstructions (2 using autograft and 10 using allograft). Modified Broström procedure was performed in 1 patient. Only 1 patient underwent total ankle arthroplasty 3 years after operation because of persistent pain. The mean BMI was 25.0 \pm 3.2 . 18 patients had a history of ankle sprain. The mean duration of symptom was $18.8 \pm 13.6$ years (Tables 1 and 2).

TAS angle improved from $82.8 \pm 2.4$ to $90.3 \pm 2.3(\mathrm{p}<0.001)$. TLS angle changed from $77.5 \pm 2.8$ to $79.4 \pm 2.7(\mathrm{p}=0.0047)$. The preoperative TT angle and postoperative TT angle were $13.4 \pm 3.6$ to $4.8 \pm 3.6$, respectively $(\mathrm{p}<0.001)$. For functional evaluation, the preoperative VAS and AOFAS-AH scores were $5.7 \pm 1.3$ and $48.0 \pm 15.8$, while the postoperative VAS and AOFAS-AH scores were 2.5 \pm 1.9 and $74.8 \pm 11.5(\mathrm{p}<0.001)$.
TABLE 1: Summarized demographic data.

\begin{tabular}{lc}
\hline parameter & Data \\
\hline Number of ankles, $\mathrm{n}$ & 21 \\
Male:female, $\mathrm{n}(\%)$ & $3(14.3 \%): 18(85.7 \%)$ \\
Side (left:right), $\mathrm{n}(\%)$ & $8(38.1 \%): 13(61.9 \%)$ \\
Age at surgery & $53.7 \pm 5.8(39-61 \mathrm{y})$ \\
Postoperative Takakura stage, $\mathrm{n}(\%)$ & \\
Stage 1 & $2(9.5 \%)$ \\
Stage 2 & $11(52.4 \%)$ \\
Stage 3A & $4(19.0 \%)$ \\
Stage 3B & $4(19.0 \%)$ \\
\hline
\end{tabular}

The mean SF-36 scale improved from $41.2 \pm 13.1$ to $66.7 \pm 14.9$ $(\mathrm{p}<0.001)$. The AOS score improved from $61.4 \pm 12.5$ to $27.5 \pm 17.8(\mathrm{P}<0.001)$.

In this study, union was achieved in all patients. 4 patients remained stage $3 \mathrm{~B}$ including the TAR one. 4 improved to stage $3 \mathrm{~A}, 11$ improved to stage 2, and 2 improved to stage 1 . (Figures 2, 3, and 4)

\section{Discussion}

For asymmetric varus type ankle arthritis, supramalleolar tibial osteotomy is an effective method. As stated above, it was usually adopted for early to midstage ankle osteoarthritis. Tanaka et al. concluded that supramalleolar osteotomy was not suitable for stage $3 \mathrm{~B}$ ankle arthritis, whereas Lee et al. thought that supramalleolar osteotomy was effective for stage $3 \mathrm{~B}$ arthritis. His study included 16 patients, and all 3 cases with $3 \mathrm{~B}$ ankle arthritis improved to stage $2[2,10]$. In our opinion, as Haraguchi et al. wrote, the osteoarthritis stage would not adequately reflect the clinical outcomes [14]. In this study, however, most patients with stage 3B varus ankle osteoarthritis achieved pain relief and better radiological manifestations. This study proved that, with proper additional procedures, supramalleolar osteotomy is an effective method for Takakura 3B ankle osteoarthritis.

The cause of varus ankle arthritis was unclear. Tanaka et al. thought it was due to lifestyle, in which people sit cross-legged or with legs tucked under the body [2]. In this study, many patients with varus ankle deformity did not have an ankle fracture history, but most of them $(85.7 \%$, $18 / 21$ ) recalled a history of old ankle sprain. Maybe it was because the weak lateral ligament resulted in varus and internal rotated dislocation of talus. With a long history of impingement at medial ankle joint and abnormal stress distribution, the varus supramalleolar deformity formed.

Knupp et al. thought that most patients with ankle arthritis present with a malaligned hinfoot [15]. Correction of hindfoot axis not only helps normalize the ankle joint load distribution but also prepares for a second surgery like ankle arthrodesis or TAR [15]. This study noticed that, with a long history of supramalleolar deformity and varus talus, many patients presented concomitant hindfoot deformity. So, in this study, near half of patients underwent calcaneal osteotomy. Patients with calcaneal osteotomy did 
TABLE 2: Radiographic and functional outcomes.

\begin{tabular}{lcccr}
\hline Parameter & preop & postop & $\mathrm{t}$ & $\mathrm{p}$ \\
\hline TAS, deg & $82.8 \pm 2.4$ & $90.3 \pm 2.3$ & 11.1 & 3.18 \\
TLS, deg & $77.5 \pm 2.8$ & $79.4 \pm 2.7$ & 9.78 & 0.0047 \\
TT, deg & $13.4 \pm 3.6$ & $4.8 \pm 3.6$ & 6.55 & $<0.001$ \\
AOFAS-AH & $48.0 \pm 15.8$ & $74.8 \pm 11.5$ & 6.37 & $<0.001$ \\
VAS & $5.7 \pm 1.3$ & $2.5 \pm 1.9$ & 6.28 & $<0.001$ \\
SF-36 & $41.2 \pm 13.1$ & $66.7 \pm 14.9$ & 7.23 & $<0.001$ \\
AOS & $61.4 \pm 12.5$ & $27.5 \pm 17.8$ & $<0.001$ \\
\hline
\end{tabular}

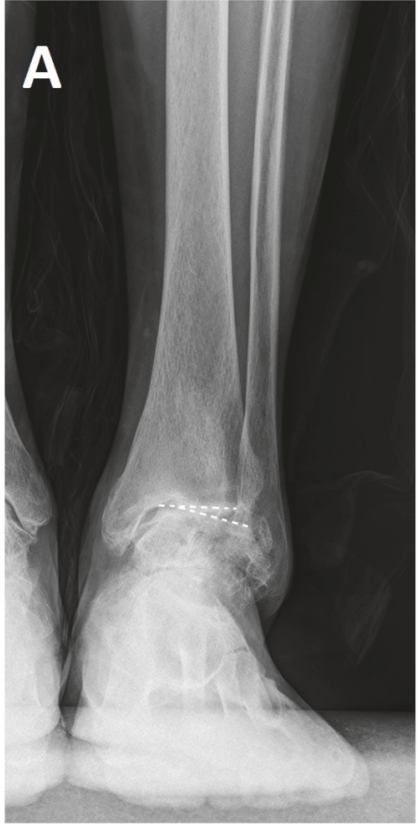

(a)

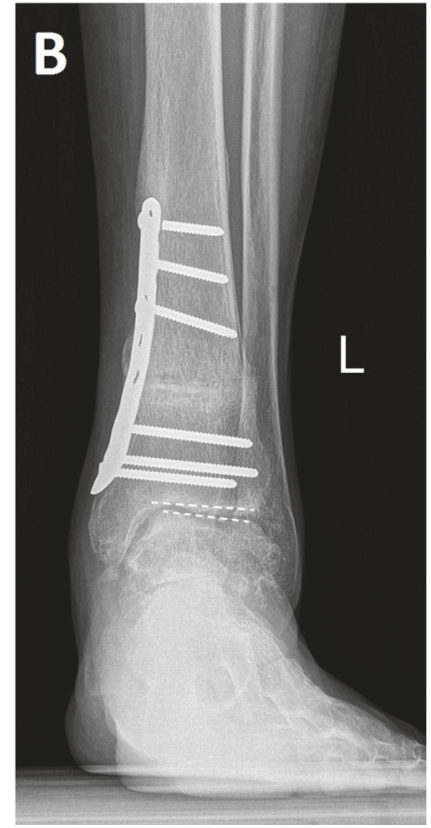

(b)

FiguRE 2: A 46-year-old woman with ankle arthritis before (a) and after (b) the supramalleolar osteotomy, the osteoarthritis stage improved to stage II.

not show any benefit compared with those without calcaneal osteotomy. The purpose of calcaneal osteotomy is to achieve normal alignment of ankle and hindfoot, which is very important for pain relief.

Tanaka thought that a $96^{\circ}$ to $98^{\circ}$ TAS angle gave better outcomes while Hintermann and Knupp recommended the TAS angle to be a little overcorrection of $92^{\circ}$ to $95^{\circ}[2,16,17]$. Pagenstert et al. overcorrected the TAS angle to be $90^{\circ}-95$ and achieved positive outcomes [11]. Hintermann et al. corrected the TAS angle to be $2^{\circ}-4^{\circ}$ valgus when the patient had medial cartilage loss [18]. In this study, the TAS angle was made in neutral position or a little overcorrected but no more than $95^{\circ}$. When facing varus ankle arthritis with large TT angle, soft tissue release is very important for it to be reduced and corrected. We do not want to achieve smaller TT angle by overcorrection of TAS.

Lee et al. and Tanaka et al. thought that when talar tilt angle was larger than $7^{\circ}$ or $10^{\circ}$, it was difficult to attain a normal ankle joint [2, 4, 10, 19]. However, in our study, most patients with preoperative TT angle larger than 10 。 achieved pain relief and change of radiographic outcomes. In this study, we performed debridement of osteophytes around ankle joint and release the soft tissue thoroughly especially the medial ligament first. We thought that the release of medial ligament was very important. The varus talus could have enough room to return to normal place under this condition with supramalleolar osteotomy, that whether or not the postoperative TT angle could return to normal matters postoperative functional outcomes. Lateral ligament reconstruction also help correct TT angle. The key point of postoperative functional improvement is how much the cartilage remains. End-stage ankle arthritis with global severe cartilage destruction is not proper for this procedure. Restoring the alignment of ankle and foot could change the abnormal distribution of stress in ankle joint and reduce pain.

This study has some limitations. First, the number of cases was small. One of the problems with this patient population is its heterogeneity. So, more studies with large 


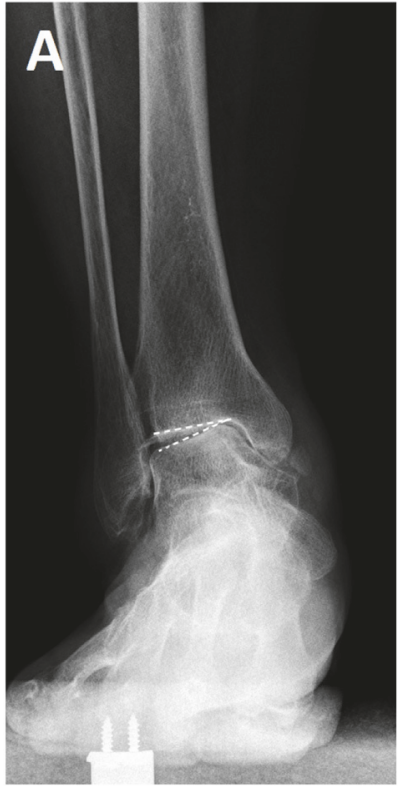

(a)

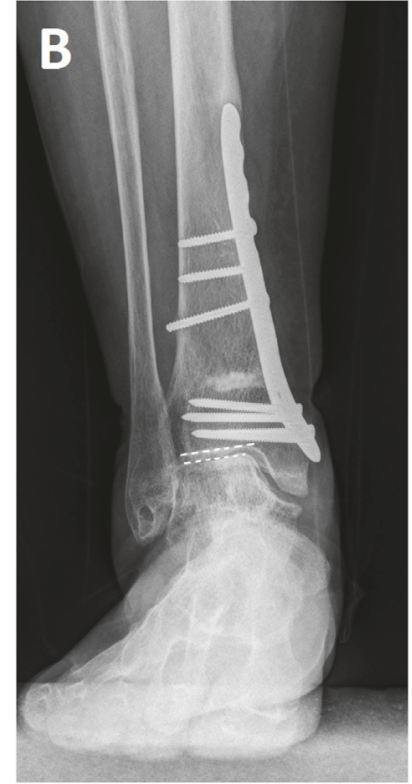

(b)

FIGURE 3: A 56-year-old woman with varus ankle arthritis before (a) and after (b) the supramalleolar osteotomy, the osteoarthritis stage improved to stage I.

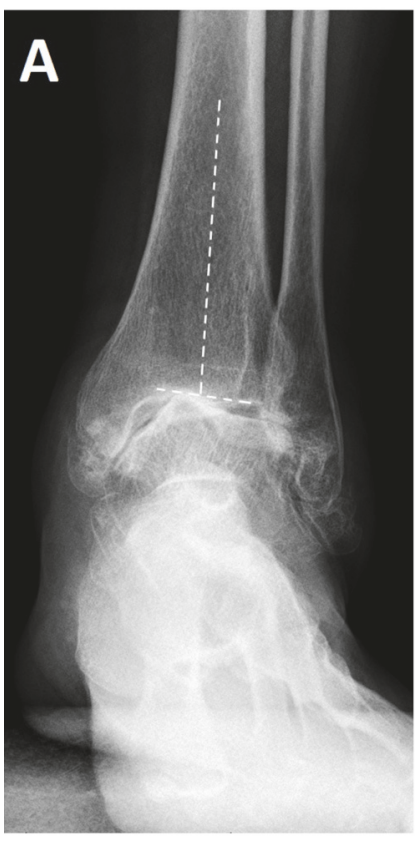

(a)

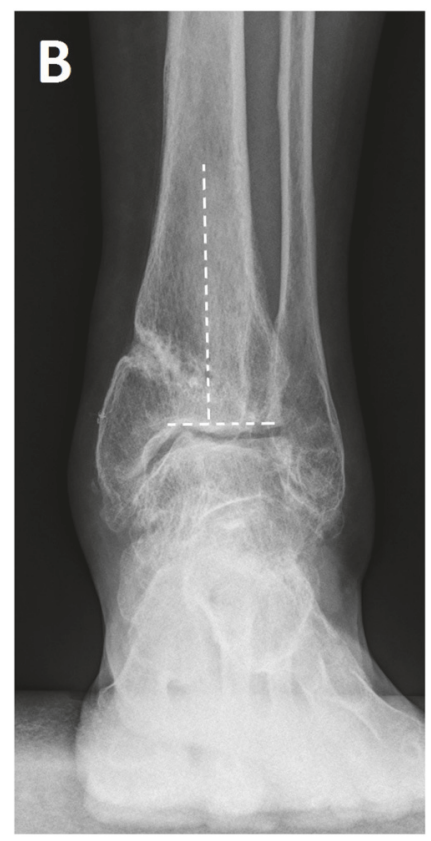

(b)

FIGURE 4: The TAS angle changed before (a) and after (b) supramalleolar osteotomy.

cases and multi center studies are necessary. Second, all patients were compared with themselves preoperatively and postoperatively. There was no blank control group. Third, the cases were assembled retrospectively in one hospital and may not be representative of other patients undergoing supramalleolar osteotomy owing to differences in ethnicity and socioeconomic status. The generalizability of the findings to different ethnical, racial, and socioeconomic populations is unknown. The strength of this study is that it shows that Takakura $3 \mathrm{~B}$ ankle arthritis is not a contraindication or relative contraindication for supramalleolar osteotomy.

In conclusion, normal alignment of ankle and hindfoot is very important for varus type ankle osteoarthritis. That whether or not the postoperative TT angle could return 
to normal matters postoperative functional outcomes. A lot of patients with large preoperative TT angles still achieved good results. Supramalleolar osteotomy can restore the malalignment of ankle joint and modify the abnormal stress distribution so as to achieve functional improvement and improve radiographic stages. Osteotomy for $3 \mathrm{~B}$ ankle arthritis can have positive outcomes and delay the time of ankle replacement or arthrodesis.

\section{Data Availability}

All data included in this study are available in the Supplementary Material.

\section{Conflicts of Interest}

The authors declare that there are no conflicts of interest.

\section{Acknowledgments}

The authors disclosed receipt of the following financial support for the research, authorship, and/or publication of this article: this study was supported by the National Natural Science Foundation of China (No. 81772372).

\section{Supplementary Materials}

The supplementary material contains detailed patients' information. (Supplementary Materials)

\section{References}

[1] Y. Takakura, Y. Tanaka, T. Kumai, and S. Tamai, "Low tibial osteotomy for osteoarthritis of the ankle. Results of a new operation in 18 patients," The Journal of Bone \& Joint Surgery (British Volume), vol. 77, no. 1, pp. 50-54, 1995.

[2] Y. Tanaka, Y. Takakura, K. Hayashi, A. Taniguchi, T. Kumai, and K. Sugimoto, "Low tibial osteotomy for varus-type osteoarthritis of the ankle," The Journal of Bone \& Joint Surgery (British Volume), vol. 88, no. 7, pp. 909-913, 2006.

[3] Z. Hongmou, L. Xiaojun, L. Yi, L. Hongliang, W. Junhu, and L. Cheng, "Supramalleolar osteotomy with or without fibular osteotomy for varus ankle arthritis," Foot \& Ankle International, vol. 37, no. 9, pp. 1001-1007, 2016.

[4] T.-K. Ahn, Y. Yi, J.-H. Cho, and W.-C. Lee, "A cohort study of patients undergoing distal tibial osteotomy without fibular osteotomy for medial ankle arthritis with mortise widening," Journal of Bone and Joint Surgery - American Volume, vol. 97, no. 5, pp. 381-388, 2015.

[5] Y. S. Kim, E. H. Park, Y. G. Koh, and J. W. Lee, "Supramalleolar osteotomy with bone marrow stimulation for varus ankle osteoarthritis: clinical results and second-look arthroscopic evaluation," The American Journal of Sports Medicine, vol. 42, no. 7, pp. 1558-1566, 2014.

[6] N. Krähenbühl, L. Zwicky, L. Bolliger, S. Schädelin, B. Hintermann, and M. Knupp, "Mid- to Long-term Results of Supramalleolar Osteotomy," Foot \& Ankle International, vol. 38, no. 2, pp. 124-132, 2017.

[7] M. Knupp, S. A. S. Stufkens, L. Bolliger, A. Barg, and B. Hintermann, "Classification and treatment of supramalleolar deformities," Foot \& Ankle International, vol. 32, no. 11, pp. 1023 1031, 2011.

[8] Y. Tanaka, “The concept of ankle joint preserving surgery. Why does supramalleolar osteotomy work and how to decide when to do an osteotomy or joint replacement," Foot and Ankle Clinics, vol. 17, no. 4, pp. 545-553, 2012.

[9] C. L. Saltzman and G. Y. El-Khoury, "The hindfoot alignment view," Foot \& Ankle International, vol. 16, no. 9, pp. 572-576, 1995.

[10] W.-C. Lee, J.-S. Moon, K. Lee, W. J. Byun, and S. H. Lee, "Indications for supramalleolar osteotomy in patients with ankle osteoarthritis and varus deformity," The Journal of Bone \& Joint Surgery, vol. 93, no. 13, pp. 1243-1248, 2011.

[11] G. I. Pagenstert, B. Hintermann, A. Barg, A. Leumann, and V. Valderrabano, "Realignment surgery as alternative treatment of varus and valgus ankle osteoarthritis," Clinical Orthopaedics and Related Research, no. 462, pp. 156-168, 2007.

[12] V. R. Prisk, C. W. Imhauser, P. F. O’Loughlin, and J. G. Kennedy, "Lateral ligament repair and reconstruction restore neither contact mechanics of the ankle joint nor motion patterns of the hindfoot," The Journal of Bone \& Joint Surgery, vol. 92, no. 14, pp. 2375-2386, 2010.

[13] X. Xu, M. Hu, J. Liu, Y. Zhu, and B. Wang, "Minimally invasive reconstruction of the lateral ankle ligaments using semitendinosus autograft or tendon allograft," Foot \& Ankle International, vol. 35, no. 10, pp. 1015-1021, 2014.

[14] N. Haraguchi, K. Ota, N. Tsunoda, K. Seike, Y. Kanetake, and A. Tsutaya, "Weight-bearing-line analysis in supramalleolar osteotomy for varus-type osteoarthritis of the ankle," Journal of Bone and Joint Surgery - American Volume, vol. 97, no. 4, pp. 333-339, 2015.

[15] M. Knupp and B. Hintermann, "Treatment of asymmetric arthritis of the ankle joint with supramalleolar osteotomies," Foot \& Ankle International, vol. 33, no. 3, pp. 250-252, 2012.

[16] B. Hintermann, M. Knupp, and A. Barg, "Joint-preserving surgery of asymmetric ankle osteoarthritis with peritalar instability," Foot and Ankle Clinics, vol. 18, no. 3, pp. 503-516, 2013.

[17] M. Knupp, L. Bolliger, and B. Hintermann, “Treatment of posttraumatic varus ankle deformity with supramalleolar osteotomy," Foot and Ankle Clinics, vol. 17, no. 1, pp. 95-102, 2012.

[18] B. Hintermann, M. Knupp, and A. Barg, "Supramalleolar osteotomies for the treatment of ankle arthritis," Journal of the American Academy of OrthopaedicSurgeons, vol. 24, no. 7, pp. 424-432, 2016.

[19] W. C. Lee, "Extraarticular supramalleolar osteotomy for managing varus ankle osteoarthritis, alternatives for osteotomy: how and why?" Foot and Ankle Clinics, vol. 21, no. 1, pp. 27-35, 2016. 


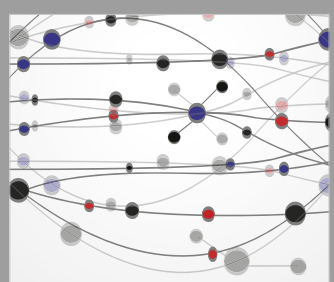

The Scientific World Journal
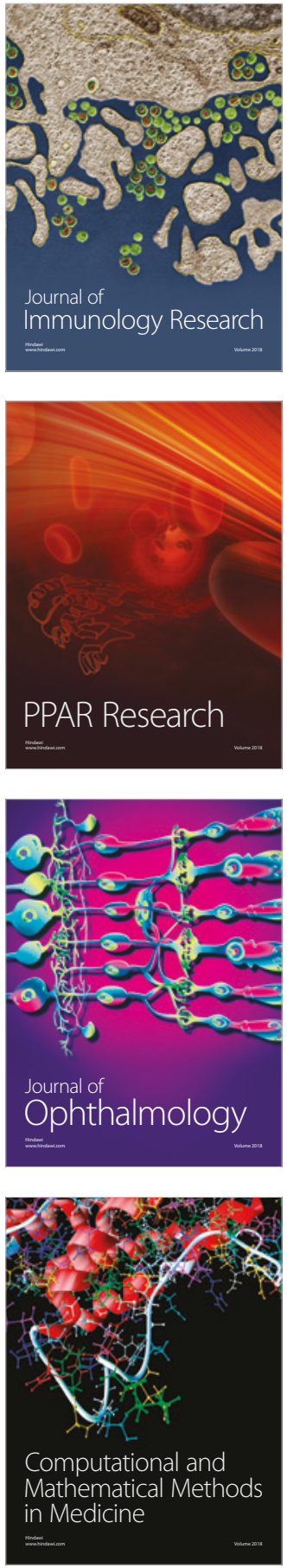

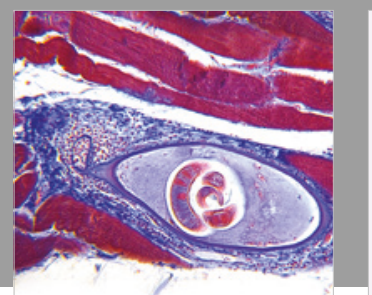

Gastroenterology Research and Practice

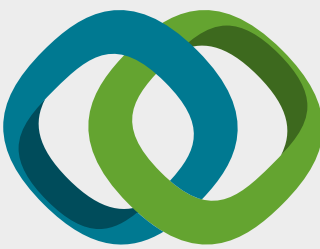

\section{Hindawi}

Submit your manuscripts at

www.hindawi.com
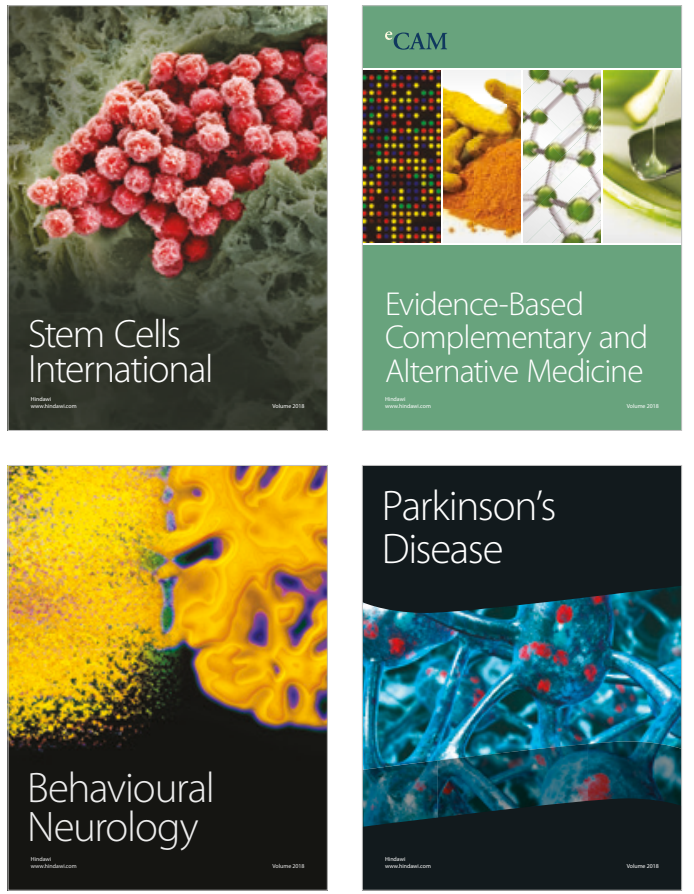

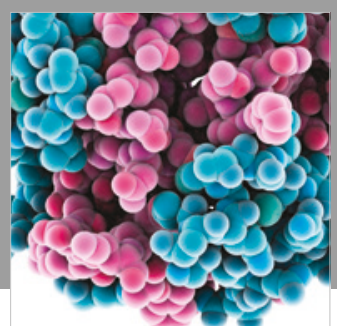

ournal of

Diabetes Research

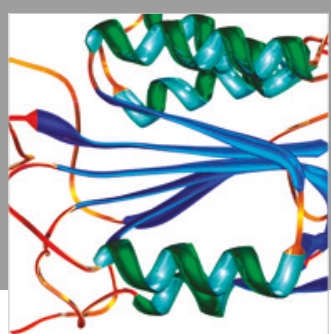

Disease Markers
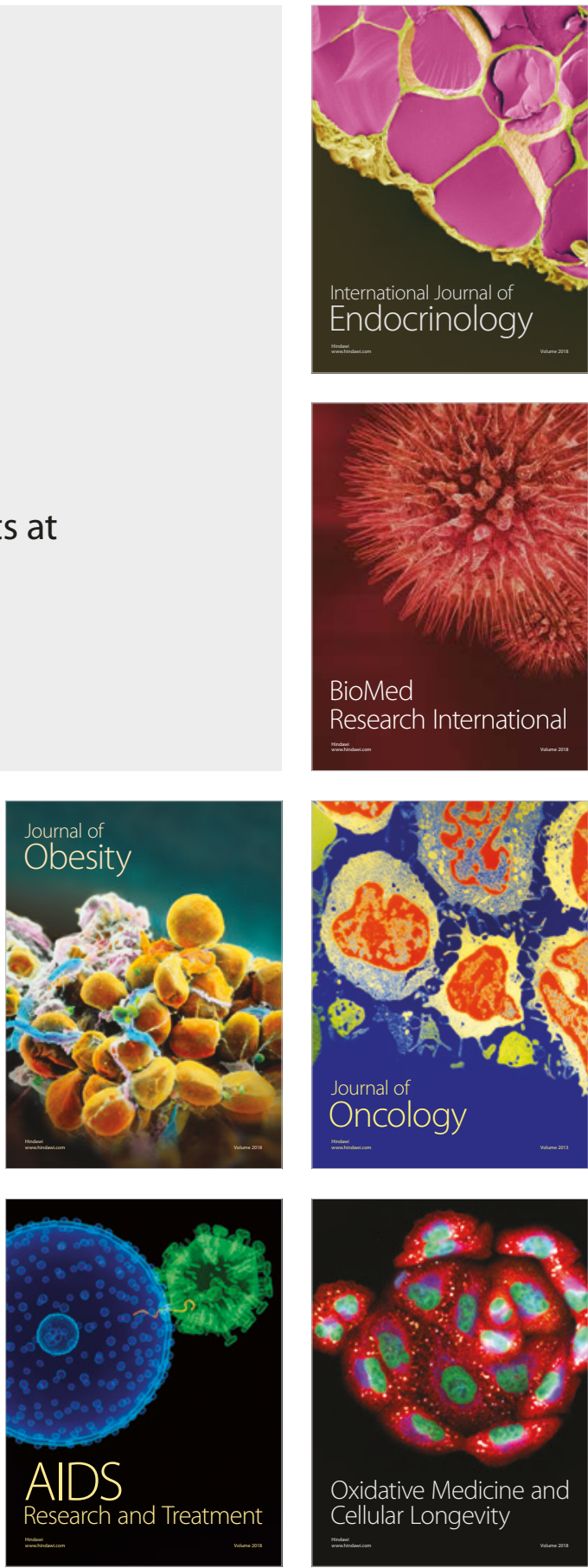